Biblioteca Universitaria

ISSN: 0187-750X

public@dgb.unam.mx

Universidad Nacional Autónoma de México

México

Hernández Ríos, Rubí; Herrera Cárdenas, Heladio; García Pérez, Jesús F.

La Biblioteca del Instituto de Investigaciones Sociales de la UNAM: su trayectoria

Biblioteca Universitaria, vol. 14, núm. 2, julio-diciembre, 2011, pp. 206-212

Universidad Nacional Autónoma de México

Distrito Federal, México

Disponible en: http://www.redalyc.org/articulo.oa?id=28521613009

- Cómo citar el artículo

- Número completo

- Más información del artículo

Página de la revista en redalyc.org

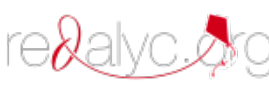

Sistema de Información Científica

Red de Revistas Científicas de América Latina, el Caribe, España y Portugal

Proyecto académico sin fines de lucro, desarrollado bajo la iniciativa de acceso abierto 


\title{
La Biblioteca del Instituto de Investigaciones Sociales de la UNAM: su trayectoria
}

\author{
Rubí Hernández Ríos, Heladio Herrera Cárdenas, \\ Jesús F. García Pérez*
}

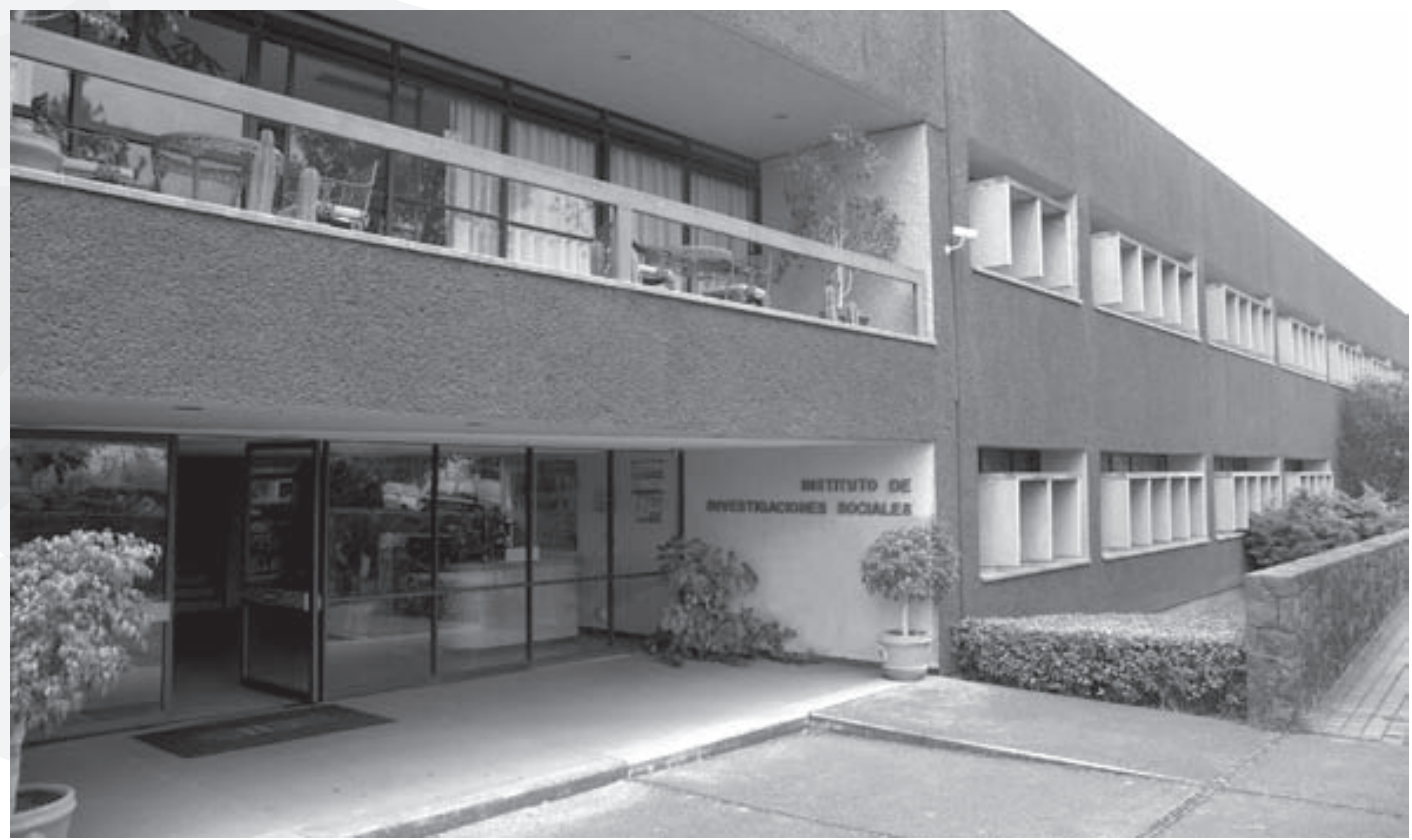

\section{Introducción}

oy en día las bibliotecas desempeñan un papel fundamental en la vida académica de sus
usuarios, por lo que es necesario que contemplen procesos que faciliten el uso adecuado
de los documentos contenidos en las mismas, de tal forma que cumplan con sus objetivos.

La Biblioteca del Instituto de Investigaciones Sociales de la Universidad Nacional Autónoma de México (IISUNAM) enfrenta el reto de proveer los servicios de información que requiere la comunidad académica de esta dependencia universitaria, así como de mantenerse actualizada en las diferentes áreas de investigación del mismo. Tiene como función primordial el apoyo documental que requieran las tareas académicas de esta dependencia universitaria y su objetivo se vincula con el propósito del propio Instituto, que es:

* Edificio del Instituto de Investigaciones Sociales, planta baja, Circuito Mtro. Mario de la Cueva, UnAM, C.U., C.P. 04510, México, D.F., México. Correos electrónicos: rubi.hernandez@unam.mx; heladio@unam.mx; garper@unam.mx 
"Llevar a cabo estudios científicos en el campo de las disciplinas sociales con el objeto de contribuir al conocimiento y solución de problemas nacionales, bajo el principio de libertad de investigación y de pluralidad teórico-metodológica, así como propagar los resultados de las investigaciones para contribuir a la difusión de investigaciones que signifiquen una aportación para las Ciencias Sociales"1.

Al pertenecer al Sistema Bibliotecario, la Biblioteca del IISUNAM presenta como objetivos generales los siguientes:

1. Planificar la prestación de servicios bibliotecarios.

2. Proporcionar servicios en toda la Universidad, garantizar que los mismos se brinden a los usuarios en forma eficiente.

3. Adecuar los servicios bibliotecarios a los avances de la ciencia y la tecnología.

4. Introducir servicios de informática y computarizados.

5. Orientar al usuario en el uso adecuado de los servicios, de tal forma que se estimulen el estudio, la investigación y la difusión de la cultura.

6. Construir acervos equilibrados.

El hecho de pertenecer a ese Sistema le ha traído como beneficios los siguientes: agilidad en la adquisición de materiales monográficos, apoyos substanciales en los procesos técnicos menores de los materiales, generación de herramientas electrónicas -como LIBRUNAM- para el apoyo de los procesos técnicos menores y para la localización de materiales monográficos en las bibliotecas que integran el Sistema, y asesorías enfocadas a mejorar los servicios internos y externos de la Biblioteca del Instituto.

El horario de servicio es de lunes a jueves de 9:00 a 15:00 hrs. y de 15:45 a 18:45 hrs. y viernes de 9:00 a 15:00 hrs. y de 15:45 a 17:30 hrs.

Universidad Nacional Autónoma de México. Instituto de Investigaciones Sociales. Historia. <http://www.iis.unam.mx/historia.html $>$ [Consulta: 18 octubre 2011].

\section{Un poco de historia}

\section{5-2011}

La Junta de Gobierno nombra a la doctora Rosalba Casas Guerrero directora del IISUNAM, quien pone énfasis en el Plan de Desarrollo y Evaluación Institucional. Con base en ello, a finales de 2006 y principios de 2007, se realizó el diagnóstico de la Biblioteca del Instituto de Investigaciones Sociales de la Universidad Nacional Autónoma de México, con el propósito de conocer en forma profunda su organización. El proceso del diagnóstico sirvió entre otros aspectos para medir el nivel de funcionamiento presentado en la biblioteca y detectar sus fortalezas y debilidades, con el fin de sugerir las formas de optimizar el funcionamiento integral de la misma. Se conformó una Comisión Ad-Hoc, integrada por representantes de la Asociación Mexicana de Bibliotecarios, A.C., el Instituto Mora, el Colegio Nacional de Bibliotecarios, FLACSO-Sede Académica de México y El Colegio de México. Dentro de los resultados, los usuarios manifestaron un alto nivel de satisfacción con la atención que les brindan los bibliotecarios.

Desde 2005, en un esfuerzo conjunto se realiza la Feria del Libro en Ciencias Sociales, que tiene como objetivo ofrecer a los investigadores y el personal académico del Instituto la posibilidad de adquirir, personalmente o bien a través de la Biblioteca, bibliografía necesaria para su trabajo docente y de investigación. Hasta 2011 se han realizado seis Ferias del Libro. Entre los servicios que proporciona podemos encontrar: consulta a bases de datos, búsquedas en Internet, préstamo a domicilio, en sala e interbibliotecario con aproximadamente 158 instituciones nacionales y 20 instituciones del extranjero; servicio de reproducción y elaboración de bibliografías para apoyar las tareas de investigación desarrolladas en el Instituto.

\section{7-2005}

Asume la dirección el doctor René Millán Valenzuela, con él se proponen modificaciones importantes para el reglamento de la biblioteca y acciones para proteger el 
Nuestras Bibliotecas. La Biblioteca del Instituto de Investigaciones Sociales de la UNAM: su trayectoria

acervo especializado. Se reorganizaron de forma interna las tareas académicas y administrativas que trajeron como beneficios a la biblioteca la integración de la Comisión de Biblioteca, conformada como lo marca el Reglamento del Sistema Bibliotecario de la UNAM, en la que el director de la dependencia funge como presidente, el coordinador de Biblioteca como secretario y participan representantes de los investigadores, de los técnicos académicos y del personal administrativo de base.

\section{9-1997}

Toma posesión como director del Instituto el doctor Ricardo Pozas Horcasitas, quien replantea y desarrolla nuevas líneas de investigación que estimulan la formación y actualización de acervos de la Biblioteca. A mediados de 1991, el doctor Pozas determina formalizar una nueva dinámica de la Biblioteca, ya que dada la riqueza documental de ésta, el incremento del personal académico del Instituto, la demanda creciente de servicios bibliotecarios, el constante desarrollo de las colecciones, entre otros aspectos, era necesario optimizar los servicios para que estuvieran acordes con la época actual y con los requerimientos de los usuarios. A finales de 1996 se termina la construcción del nuevo edificio para el Instituto, ubicado en la Ciudad de la Investigación en Humanidades -Circuito Mtro. Mario de la Cueva, Zona Cultural, c.U.-, sede actual del IISUNAM.

\section{0-1980}

En la década de los 70's se asigna al Instituto un pequeño local en la antigua Escuela de Medicina; para esta época la biblioteca contaba con un fondo aproximado de 8000 volúmenes sobre temas especializados en ciencias sociales.

En 1975 se comienza a publicar el Boletín Bibliográfico, conteniendo el análisis de las publicaciones periódicas de reciente adquisición.

A finales de los 70's el Instituto se ubicaba en la Torre I de Humanidades y por la demanda de un espacio físico más amplio para todas las áreas se traslada a la Torre II de Humanidades.
En 1980 celebra el contrato de cooperación para integrarse a la Red de Consulta del Servicio Público de Información Estadística y Geográfica, perteneciente al INEGI. En 1984 el acervo tenía 20,000 volúmenes monográficos y 400 títulos de publicaciones periódicas. En 1985 se modifica el reglamento interno del Instituto, la Sección de Información, Documentación y Biblioteca se divide y se forma un Departamento de Documentación y una Coordinación Académica de Biblioteca.

\section{0-1960}

A mediados de la década de los 50's la Biblioteca se ubicó en la casa núm. 3 de la calle de Licenciado Verdad. En 1966 toma posesión como director del Instituto el doctor Pablo González Casanova, quien lo reorganiza. Es así cómo se originaron tres áreas especializadas que funcionaron de manera permanente: Sección de Información, Documentación y Biblioteca; Sección de Muestreo, Programación, Cálculo y Análisis, y, Sección de Publicaciones.

En el mismo año ya se observa como un órgano importante del Instituto a la "Sección de Información, documentación y Biblioteca", y es cuando empieza haber preocupación porque en ella trabaje un especialista en documentación y archivos; si bien la persona que estuvo al frente no era un especialista en Bibliotecología, según la información recabada, estaba capacitada para realizar las tareas primordiales dentro de esta Sección.

\section{0-1940}

Desde su fundación, en 1930, el Instituto se organiza en cuatro áreas temáticas: Instituciones sociales; Población; Instituciones económicas; Instituciones jurídicas y políticas.

La Biblioteca del Instituto contaba a finales de 1940 con más de 3000 volúmenes, constituyendo un valioso auxiliar para su personal de investigación. Por estas fechas se ubicó al Instituto en el último piso del edificio ocupado por la Escuela de Economía, en la calle de República de Cuba núm. 92, que posteriormente pasó a ocupar dos estancias en la planta baja del antiguo edificio principal sede de la Universidad, en la calle de Justo Sierra núm.16. 


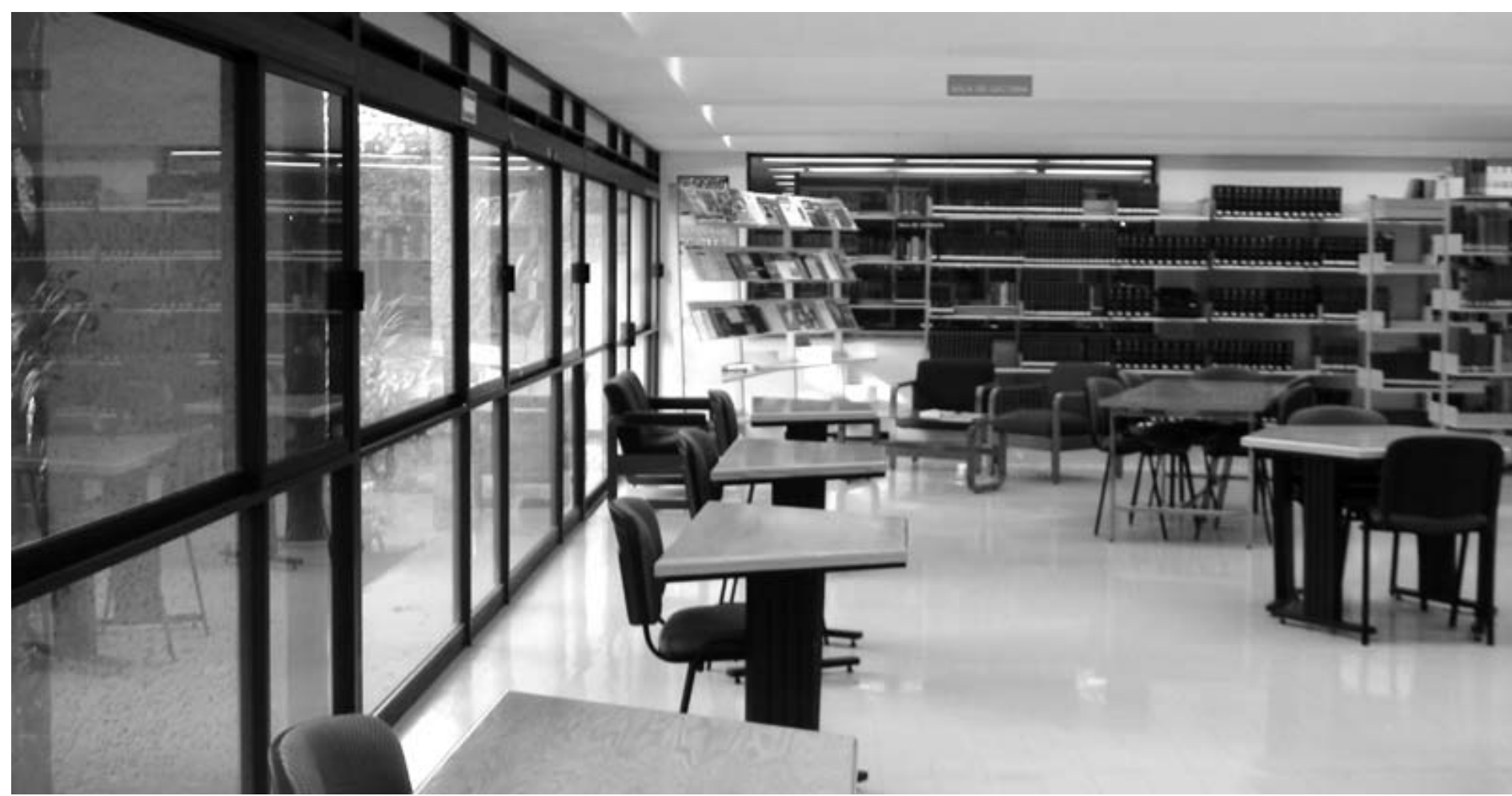

Investigar y estudiar para el beneficio social de México

La anterior es la frase que se lee en la placa conmemorativa del 75 aniversario del Instituto que está en la entrada, la cual pocos se detienen a leer y sin embargo allí sigue, muda testigo de los cambios que han sucedido a lo largo de seis años. Recientemente fue presentado el Segundo Informe de Labores de la directora del Instituto, donde menciona la relación existente entre los resultados presentados en el Informe Mundial sobre las Ciencias Sociales 2010: Brechas del conocimiento y las tareas realizadas en el Instituto; cómo no voltear y reflexionar sobre la placa que está en la entrada, es ésta nuestra carta de presentación como instituto desde hace más de ochenta años, hay entonces un estrecho vínculo entre los resultados mundiales y lo que a lo largo de ocho décadas se ha investigado y estudiado.

A finales del año anterior fue publicado ese informe elaborado por la UNESCO, donde se mencionan las diferentes situaciones a las que se enfrenta la sociedad en estos tiempos, con las cuales se identifica plenamente. En ese testimonio se presentan los principales proble- mas y aspectos de riesgo en las disciplinas sociales, "el documento sostiene que dados los cambios globales y los cambios que afectan a todas las sociedades, el conocimiento de las ciencias sociales es más crucial que nunca antes". ${ }^{2}$ Aquí cabe mencionar las áreas de investigación que se desarrollan en nuestro Instituto:

\section{Estudios agrarios.}

2. Población y estudios demográficos.

3. Estudios urbanos y regionales.

4. Estudios de la educación y la ciencia.

5. Instituciones políticas.

6. Actores y procesos sociales.

7. Sociedad y cultura.

En cada área temática existen diversas líneas de investigación para las cuales la Biblioteca juega un papel fundamental debido a que es la principal proveedora y alimentadora de información en los proyectos de cada línea.

2 CAsas Guerrero, Rosalba. Segundo informe de labores 20102011. 
Nuestras Bibliotecas. La Biblioteca del Instituto de Investigaciones Sociales de la UNAM: su trayectoria

Uno de los principales ejes en el Plan de Desarrollo del Instituto es mantener un vínculo entre el investigador y la información, por tal motivo es menester proporcionar a la comunidad académica las herramientas necesarias para cubrir la demanda de cada necesidad de información.

\section{Desarrollo de colecciones}

De acuerdo con estadísticas proporcionadas por la Dirección General de Bibliotecas (DGB) de la UNAM, en la actualidad la Biblioteca del Instituto resguarda:

- Acervo:

- Número de títulos $=28049$.

- Número de ejemplares $=35903$ volúmenes.

- Material publicado por INEGI $=4239$ volúmenes.
- Revistas:

- Número de títulos $=499$.

- Número de ejemplares $=22043$.

- Folletos: Más de 4567.

- Bases de datos en CD: títulos = 153; volúmenes $=323$.

- Videos: 107.

La gráfica 1 presenta la tendencia de crecimiento de la adquisición de material bibliográfico en los últimos ocho años.

Todos los servicios están encaminados a satisfacer primordialmente las necesidades de información del cuerpo académico de investigadores que labora en el Instituto. Cuenta con estantería mixta, es decir, abierta para el personal académico del IIS y cerrada para el público en general

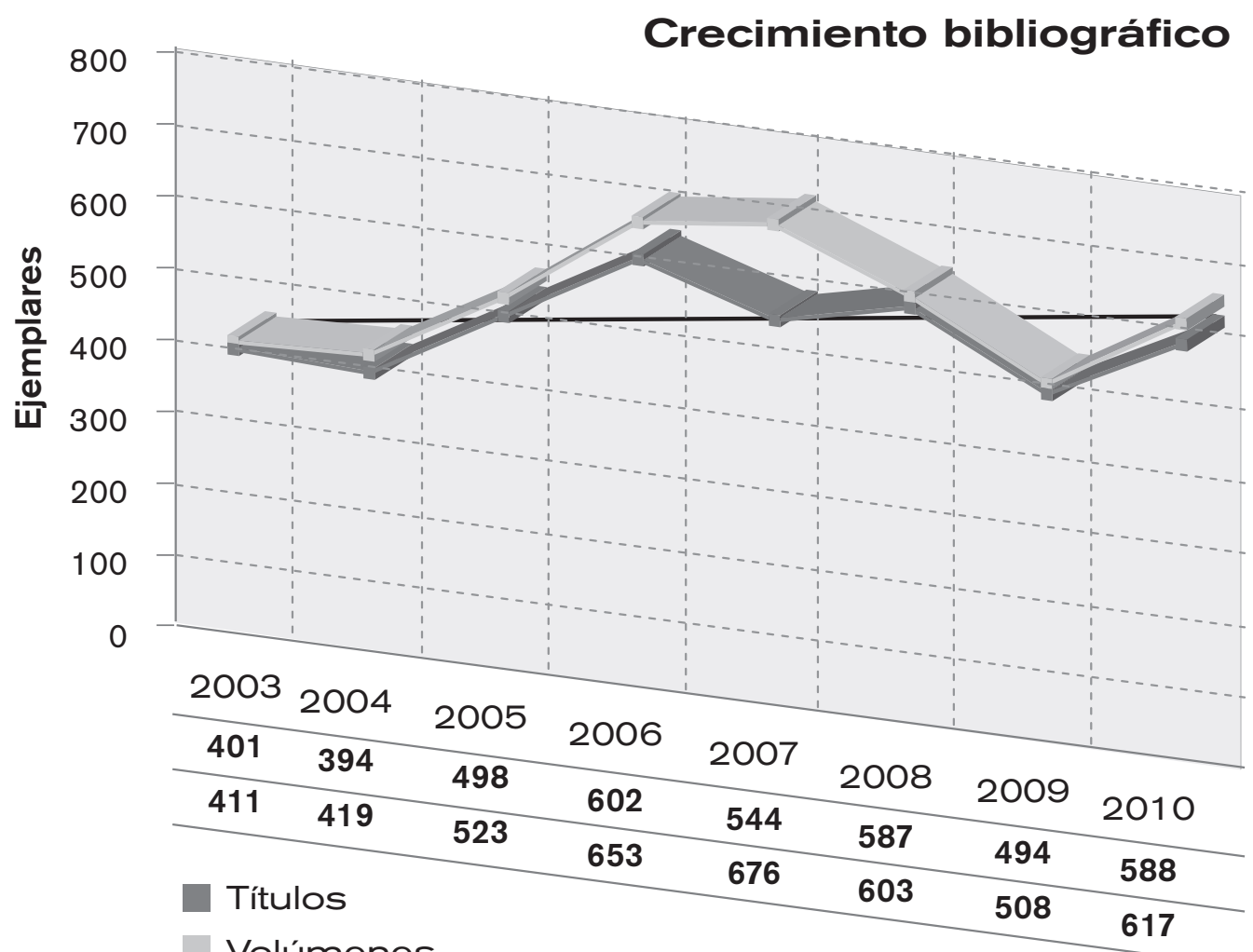

Gráfica 1. Tendencia de crecimiento 


\section{Selección y adquisición de material documental}

La Biblioteca del IISUNAM selecciona los materiales documentales pertinentes con base en las líneas de investigación del Instituto y canaliza los pedidos con los diferentes proveedores la Dirección General de Bibliotecas de la UnAM, autorizados y no autorizados, respetando los procedimientos establecidos por esta dirección.

La selección de los materiales debe apegarse a las líneas temáticas de investigación del Instituto.

En el punto correspondiente a la donación de monografías, se toman las siguientes acciones:

- Antes de aceptar una donación, se analiza el tipo y la temática del material que se quiere donar.

- Al llegar el material donado, se revisa con la finalidad de evitar duplicidad y que ingrese material innecesario, de acuerdo con las líneas de investigación desarrolladas.

- Los materiales duplicados o que no corresponden a las temáticas se separan para canalizarlos a otras instancias.

- Asimismo, el buen control de la selección de materiales se ha visto beneficiado por la implantación de un servicio informativo, que consiste en la compilación de catálogos de proveedores; igualmente, se estudian las temáticas presentadas y con base a ello los materiales se distribuyen a los coordinadores de área con la finalidad de que seleccionen los de su interés.

- Se implementó un seguimiento de los materiales solicitados para su adquisición, el cual consiste en remitir al investigador el estado que guardan los materiales que ha solicitado: cuándo se tramitó el pedido, qué van a surtir y qué es lo que no será surtido, cuándo ingresa a la biblioteca el material, entre otros aspectos. Este seguimiento se ha implementado a través de la puesta en marcha del Sistema Aleph.

Por lo que respecta al canje:

- Se establece contacto estrecho con el departamento encargado de editar la Revista Mexicana de Sociología, con la finalidad de estar al día tanto la biblioteca como ese departamento en el control del canje.

En el aspecto del proceso técnico menor que se realiza a los documentos, la DGB realiza los procesos técnicos de manera centralizada.

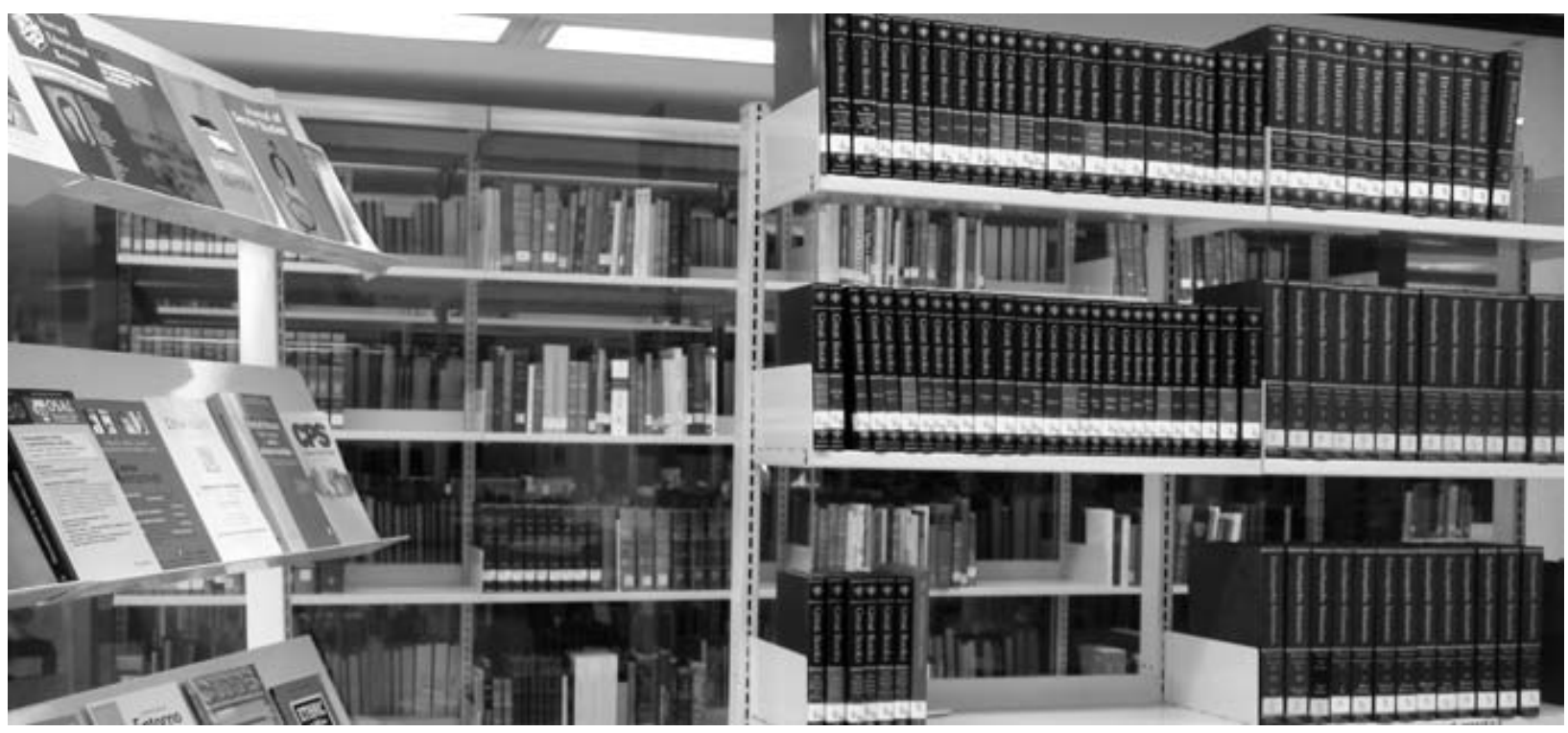


Nuestras Bibliotecas. La Biblioteca del Instituto de Investigaciones Sociales de la UNAM: su trayectoria

Al adquirir el material se canalizan fotocopias de las portadas, contraportadas e índices del material al Departamento de Procesos Técnicos de la DGB de la UNAM. Internamente, la biblioteca del IISUNAM realiza el proceso técnico menor de los materiales, consistente en: sellado, etiquetado, marcado de esquineros y tarjetas de control.

\section{Software, hardware y redes}

El campo tecnológico, el software y hardware, así como el uso de redes, cada vez más toman gran importancia dentro de las áreas de la información, éstas representan una interesante combinación entre aplicaciones, sistemas y teorías tradicionales del manejo de información ordenada y estructurada. Asimismo, representan amplias oportunidades para el campo de la investigación al extender y mejorar el acceso a nuevas formas de acercarnos al conocimiento, en nuestro caso especialmente en áreas afines a las ciencias sociales; actualmente la biblioteca cuenta con la infraestructura necesaria de cómputo, hardware y software, que le permite el óptimo desarrollo de actividades especializadas.

\section{Personal de la Biblioteca}

Actualmente, el personal está conformado de la siguiente manera:

\begin{tabular}{|c|c|}
\hline Área & $\begin{array}{l}\text { Preparación } \\
\text { académica }\end{array}$ \\
\hline $\begin{array}{l}\text { Coordinador académico de la } \\
\text { Biblioteca }\end{array}$ & Doctorado \\
\hline Selección y adquisición & Pas. maestría \\
\hline Consulta a bases de datos & Pas. maestría \\
\hline Revistas & Pas. maestría \\
\hline Proceso físico y servicios & $\begin{array}{l}\text { Personal administrativo } \\
\text { de base }\end{array}$ \\
\hline
\end{tabular}

El Instituto es la entidad académica más antigua del Subsistema de Humanidades y con sus 90 investigadores se coloca en el segundo lugar en tamaño de dicho Subsistema, después del Instituto de Investigaciones Filológicas. El perfil de la planta de los investigadores refleja una composición diversa por grupos de edad, por lo que coexisten diversos conjuntos generacionales.

La Biblioteca del IISUNAM, con más de 60 años de historia, se enmarca dentro de las bibliotecas universitarias especializadas en combinación con las tecnologías de información y comunicación, que juegan un papel importante para el desarrollo de colecciones, servicios y espacios físicos. os

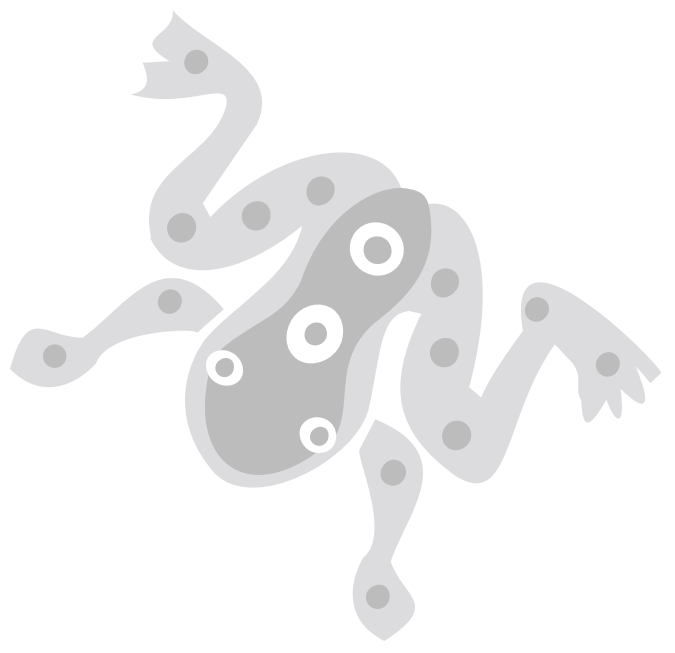

\section{Obras Consultadas}

Reglamento de la biblioteca. México: unAm, Instituto de Investigaciones Sociales, 2003. 28 p.

Universidad Nacional Autónoma de México. Instituto de Investigaciones Sociales. Segundo informe de labores agosto 2010 - agosto 2011. Rosalba Casas Guerrero. < http://www.iis.unam. $\mathrm{mx} /$ pdfs/inf_labores_1011.pdf > [Consulta: 18 octubre 2011]. 